\title{
Immune Response to Type III Group B Streptococcal Polysaccharide-Tetanus Toxoid Conjugate Vaccine
}

\author{
Dennis L. Kasper, ${ }^{\star}$ Lawrence C. Paoletti, ${ }^{\star}$ Michael R. Wessels, ${ }^{\star \ddagger}$ Hilde-Kari Guttormsen, ${ }^{\star}$ Vincent J. Carey, ${ }^{\star}$ \\ Harold J. Jennings, ${ }^{\S}$ and Carol J. Baker\| \\ *Channing Laboratory, Brigham and Women’s Hospital, and ${ }^{\ddagger}$ Division of Infectious Diseases, Beth Israel Hospital, Harvard Medical \\ School, Boston, Massachusetts 02115; ' Institute for Biological Sciences, National Research Council of Canada, Ottawa, Ontario, K1A 0R6,

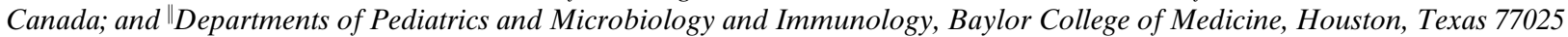

\begin{abstract}
Group B Streptococcus (GBS) is an important perinatal pathogen. Because transplacentally acquired maternal antibodies to the GBS capsular polysaccharides (CPS) confer protection, prevention of infant disease may be possible after immunization of women. Unfortunately, the purified CPS of GBS are only variably immunogenic in adults; therefore to enhance immunogenicity we have designed and developed a CPS-protein conjugate vaccine. The lability of a conformationally dependent epitope on the III CPS containing a critical sialic acid residue was important to consider in vaccine design. 100 women were randomized to receive GBS type III CPS-tetanus toxoid conjugate (III-TT) vaccine at one of three doses; unconjugated GBS type III CPS; or saline. Serum samples were obtained before immunization and 2, 4, 8, and 26 wk thereafter, and specific antibody to type III CPS was measured. Vaccines were well tolerated. In sera from recipients of the highest dose of III-TT, CPS-specific IgG levels rose from a geometric mean of 0.09 $\mu \mathrm{g} / \mathrm{ml}$ before immunization to $4.53 \mu \mathrm{g} / \mathrm{ml} 8 \mathrm{wk}$ later, whereas levels in recipients of unconjugated type III CPS rose from $0.21 \mu \mathrm{g} / \mathrm{ml}$ to $1.41 \mu \mathrm{g} / \mathrm{ml}$. Lower doses resulted in lower antibody levels. $\mathrm{A} \geq 4$-fold rise in antibody concentration was achieved in $90 \%$ of recipients of III-TT compared with $50 \%$ of those that received III CPS $(P=0.0015)$. Antibodies evoked by the conjugate vaccine recognized a conformationally dependent epitope of the III-CPS, promoted opsonophagocytosis and killing of GBS, and, after maternal immunization, protected neonatal mice from lethal challenge with type III GBS. We conclude that directed coupling of type III GBS polysaccharide to a carrier protein yielded a conjugate vaccine with preserved expression of a highly labile conformational epitope involving sialic acid and enhanced immunogenicity compared with uncoupled
\end{abstract}

This work was presented in part at the Annual Meeting of the Infectious Diseases Society of America, 16-18 September 1995, San Francisco, CA.

Address correspondence to Dennis L. Kasper, Channing Laboratory, 181 Longwood Avenue, Boston, MA 02115. Phone: 617-5252280; FAX: 617-731-1541; E-mail: dkasper@warren.med.harvard.edu Received for publication 1 July 1996 and accepted in revised form 13 September 1996.

J. Clin. Invest.

(C) The American Society for Clinical Investigation, Inc. 0021-9738/96/11/2308/07 \$2.00

Volume 98, Number 10, November 1996, 2308-2314
CPS. (J. Clin. Invest. 1996. 98:2308-2314.) Key words: maternal vaccination - neonatal infections - glycoconjugates $\bullet$ women's health $\bullet$ perinatal infections • group B streptococci

\section{Introduction}

Group B Streptococcus (GBS) ${ }^{1}$ is the most common cause of serious infections in newborns and young infants, with a casefatality ratio of $5-8 \%$. It is also a prominent cause of peripartum maternal infections, causing an estimated 50,000 cases annually (1). In addition, recent reports have documented the importance of GBS as a cause of infection in adults with certain underlying medical conditions (2-4). The attack rate for GBS disease among neonates has been estimated at 1.8 cases per 1,000 live births (5) and the adult rate of infection at four cases per 100,000 population (2).

Differences among GBS serotypes are based on the organisms' capsular polysaccharide (CPS) antigens. Capsular type III is most often associated with meningitis and infant infections with onset more than $7 \mathrm{~d}$ after birth (1). Because antibodies to CPS confer protective immunity to GBS infection both in experimental animals (6) and in human infants (7), a logical approach to the prevention of GBS perinatal infections is a vaccine that elicits CPS-specific antibodies.

Previous vaccine trials tested the immunogenicity of GBS CPS in adult volunteers (8). Subjects with low levels of preexisting serum antibodies to the immunizing CPS had suboptimal increases in antibody concentrations after immunization. About $40 \%$ of volunteers with low levels of antibody before immunization responded to type Ia CPS, $60 \%$ to type III, and $88 \%$ to type II. The response rate was $63 \%$ among women vaccinated with GBS type III CPS during the third trimester of pregnancy. Among subjects who responded, maternal IgG efficiently crossed the placenta (9). These observations suggested that maternal vaccination might be an effective approach to the prevention of perinatal GBS infection if the immunogenicity of the organism's CPS could be improved.

Immune responses to CPS antigens have been enhanced in animals by the coupling of CPS to carrier proteins (10). Covalent conjugation of carbohydrates to proteins can be achieved using either random or targeted sites on the polysaccharide. In prior studies we have shown that protective antibodies against the type III CPS recognize an epitope which is conformationally dependent both on the sialic acid residues and on the

1. Abbreviations used in this paper: CPS, capsular polysaccharide; GBS, group B Streptococcus; GMC, geometric mean concentration; III-TT, type III CPS tetanus toxoid conjugate; TT, tetanus toxoid. 
chain length of the CPS (11). To preserve this conformational epitope, we created a target site on the CPS for the covalent attachment of proteins. Herein we present an evaluation of the safety and immunogenicity of GBS type III CPS-tetanus toxoid conjugate vaccine (III-TT) in healthy, nonpregnant women.

\section{Methods}

\section{Preparation of vaccines}

Type III CPS (III CPS) and III-TT were prepared and their compositions analyzed as described previously (12). In brief, III CPS antigen was purified from culture supernatants of GBS strain M781 (12). III-TT was prepared by coupling of III CPS (average $M_{\mathrm{r}}, \sim 220,000$ ) to monomeric TT. Before coupling, III CPS was oxidized chemically to create aldehyde groups on $26 \%$ of the sialic acid residues. The degree of oxidation of III CPS was verified by gas-liquid chromatography/mass spectrometry. The terminal aldehyde groups created on III CPS were available for covalent coupling to TT by reductive amination with sodium cyanoborohydride. The conjugate vaccine was purified by gelfiltration chromatography and dialyzed against deionized water. The purified III-TT vaccine was composed of $56 \%$ (wt/wt) III CPS and $44 \%$ (wt/wt) TT. Placebo (lot No. 02-93) consisted of $0.9 \%$ saline containing $0.01 \%$ thimerosal provided by the National Institute of Allergy and Infectious Diseases contract number 45196.

\section{Safety evaluation of bottled $G B S$ vaccines}

GBS type III-TT vaccine (lot No. 91-1) and GBS type III CPS vaccine (lot No. 91-8s) passed tests conducted by DynCorp-PRI, Rockville, $\mathrm{MD}$, in accordance with the relevant regulations of the Food and Drug Administration, i.e., title 21, sections 610.11 (general safety); 610.12 (microbial sterility); and 610.13 (pyrogenicity).

\section{Subjects}

100 healthy nonpregnant women between 18 and $40 \mathrm{yr}$ of age were enrolled in one of two studies after giving written informed consent. The studies were approved by the Institutional Review Board for Human Subject Research at Baylor College of Medicine. Participants were required to have a negative serum pregnancy test, a normal limited physical examination, no acute or chronic illnesses, and no history of tetanus immunization within the previous $5 \mathrm{yr}$. Study 1 included 25 Caucasians, 4 African-Americans, and 6 Hispanics. Subjects were randomized to receive one of the two vaccines ( $n=15$ per group) or placebo $(n=5)$. The vaccines administered were III-TT $(58 \mu \mathrm{g}$ of polysaccharide per dose) and uncoupled III CPS (50 $\mu \mathrm{g}$ per dose). Study 2 included 48 Caucasians, 7 African-Americans, 7 Hispanics, and 3 Asians. In this study, subjects were randomized to receive one of four vaccine regimens $(n=15$ per group) or placebo $(n=5)$. The vaccines administered were III-TT $(58,14.5$, or $3.6 \mu \mathrm{g}$ of polysaccharide per dose) and III CPS (50 $\mu \mathrm{g}$ per dose). Volunteers in both studies received $0.5 \mathrm{ml}$ of a coded preparation as an intramuscular injection into the deltoid region. Subjects and study personnel assessing systemic and local reactogenicity after the injection were blinded as to vaccine administered. Subjects were interviewed and examined 24 and $48 \mathrm{~h}$ after vaccination, and their symptoms as well as their local reactions and temperature were recorded daily for $8 \mathrm{~d}$. Complete blood counts (CBC) and differentials as well as 20 serum chemistries were obtained for participants of study 1 before and $48 \mathrm{~h}$ after vaccination. Serum samples were obtained from all subjects immediately before vaccination and 2, 4, 8, and $26 \mathrm{wk}$ thereafter.

\section{Serological methods}

Specific IgG (total and subclass), IgM, and IgA to III CPS were measured by ELISA; III CPS covalently linked to HSA (III-HSA) was used as the coating antigen (13). Antibody values determined by ELISA correlate well with values determined by radioactive antigenbinding assay, but the ELISA has been shown to offer advantages, including greater sensitivity. The lower limits of detection in the ELISA were $0.05 \mu \mathrm{g}$ of III CPS-specific $\mathrm{IgG} / \mathrm{ml}$ of serum and $0.08 \mu \mathrm{g}$ of III CPS-specific IgA/ml. III CPS-specific IgM was measured as a dilution titer. The IgG response to the TT component of III-TT in sera obtained from study 1 volunteers (excluding placebo group) before and 8 wk after vaccination was quantified by ELISA (14) in the laboratory of Dr. George Siber at the Massachusetts State Department of Health.

\section{Binding specificity of III-TT-induced antibodies}

The relative functional binding affinity of III-TT-induced antibodies for III CPS of various chain lengths $(11,15)$ was determined by an inhibition ELISA in which wells were coated with III-HSA (13). Native III CPS $\left(>150,000 M_{\mathrm{r}}\right)$, III CPS of 25 pentasaccharide repeating units $\left(25,500 M_{\mathrm{r}}\right)$, a 1-pentasaccharide type III oligosaccharide $\left(1,022 M_{\mathrm{r}}\right)$, and HSA were used as inhibitors of antibody binding in the ELISA. Five randomly chosen sera obtained 8 wk after vaccination were tested individually in this assay.

The relative functional binding affinity for the native III CPS of III-TT induced antibodies was compared by ELISA inhibition to that of III CPS-induced antibodies. Five sera obtained 8 wk after vaccination with either III-TT or III CPS were tested individually.

\section{Functional activity of vaccine-induced antibodies}

In vitro. Sera were collected and stored at $-80^{\circ} \mathrm{C}$ to preserve complement activity. The ability of III CPS- and III-TT-induced sera to opsonize GBS cells for killing by human peripheral blood polymorphonuclear leukocytes was determined in an in vitro opsonophagocytosis assay (9). Sera obtained before and at 4 wk after vaccination from 45 randomly selected recipients of III-TT (all doses, $n=30$ ) and III CPS $(n=15)$ were tested at a single 1:10 dilution. Results were expressed as the difference between the number of CFU of GBS at initiation of the experiment and that after $40 \mathrm{~min}$ of incubation. The correlation coefficients $(r)$ between the $\log _{10}$ decrease in CFU and the III CPS-specific antibody concentration in these 45 sera were determined.

In vivo. The mouse maternal vaccination/neonatal challenge model of GBS infection (16) was used for in vivo assessment of the protective ability of selected sera containing vaccine-induced antibodies. A standard human reference serum pool for serotype III was generated by combining sera from five women immunized with III-TT; this reference serum contained $83.5 \mu \mathrm{g}$ of III CPS-specific IgG/ml (13). $2 \mathrm{~d}$ before delivery of their offspring, four pregnant mice (CD-1 mice at 18-19 d of gestation) were given a single intraperitoneal injection of $0.5 \mathrm{ml}$ of the standard human reference serum pool, one pregnant dam received $0.5 \mathrm{ml}$ of human serum containing $<0.3 \mu \mathrm{g}$ of III CPSspecific antibody $/ \mathrm{ml}$, and two pregnant dams received $0.5 \mathrm{ml}$ of rabbit serum to III-TT (12) containing 1,500 $\mu \mathrm{g}$ of III CPS-specific antibody $/ \mathrm{ml}$. At $1 \mathrm{~d}$ of life, each pup was challenged with $5 \times 10^{4}-6 \times 10^{4} \mathrm{CFU}$ of GBS type III strain M781 $\left(1,000 \mathrm{LD}_{50}\right)$. Survival was assessed after $48 \mathrm{~h}$.

Statistical analysis. Because the two studies used the same vaccine lots, performance statistics for each vaccine preparation were derived from measurements for all recipients of that preparation. Natural logarithms of antibody concentrations were assumed to be normally distributed. Antibody concentrations that were reported to be lower than the limit of detection $(0.05 \mu \mathrm{g} / \mathrm{ml})$ are "left censored." Maximum likelihood estimation of geometric mean antibody concentrations (and associated confidence intervals) based on the results of experiments in which some observations are censored was accomplished with the PROC LIFEREG program (SAS Institute, Cary, NC) (17). Vaccine response distributions (as opposed to means) were illustrated with the reverse cumulative distribution plot (18). Geometric mean distributions of individual postvaccination fold-rise values in antibody concentrations were compared nonparametrically with a modification of the Wilcoxon rank-sum statistic for use in repeated-measures experiments (19). Differences between proportions of vaccinees exhibiting certain response characteristics (fold-rise value of $\geq 4$, or 8 -wk concentration of $>1 \mu \mathrm{g} / \mathrm{ml}$ ) were assessed by Fisher's exact test (20). 
Table I. Reactogenicity to Group B Streptococcal Vaccines

\begin{tabular}{|c|c|c|c|c|c|c|c|c|c|}
\hline \multirow[b]{2}{*}{ Vaccine, dose } & \multirow{2}{*}{$\begin{array}{l}\text { No. of } \\
\text { recipients }\end{array}$} & \multicolumn{4}{|c|}{ Pain* } & \multicolumn{4}{|c|}{ Redness and swelling } \\
\hline & & 0 & 1 & 2 & 3 & 0 & 1 & 2 & 3 \\
\hline$\mu g$ & & \multicolumn{4}{|c|}{$\%$} & \multicolumn{4}{|c|}{$\%$} \\
\hline \multicolumn{10}{|l|}{ III-TT } \\
\hline 58 & $30^{\ddagger}$ & 37 & 33 & 30 & 0 & 73 & 17 & 10 & 0 \\
\hline 14.5 & 15 & 33 & 33 & 33 & 0 & 86 & 7 & 0 & 7 \\
\hline 3.6 & 15 & 67 & 27 & 7 & 0 & 73 & 27 & 0 & 0 \\
\hline III CPS, 50 & 30 & 60 & 27 & 13 & 0 & 97 & 0 & 3 & 0 \\
\hline Saline, $\mathrm{NA}^{\S}$ & 10 & 60 & 10 & 30 & 0 & 100 & 0 & 0 & 0 \\
\hline
\end{tabular}

$* 0$, no pain; 1 , tender; 2 , sore with movement; 3 , unable to move arm. ${ }^{0}$, none; $1,1-3 \mathrm{~cm} ; 2,3-5 \mathrm{~cm} ; 3,>5 \mathrm{~cm}$. ${ }^{\ddagger}$ One subject $(3 \%)$ had a temperature of $100.3^{\circ} \mathrm{F} 24 \mathrm{~h}$ after immunization. ${ }^{\S}$ Not applicable.

\section{Results}

\section{Safety}

Overall, both III-TT (at any dose) and III CPS were well tolerated, with minimal reactogenicity. The majority of women receiving III-TT at any dose experienced only tenderness or no symptoms at the site of injection (Table I). Similarly low rates of reactogenicity were seen in recipients of III CPS or saline. Most women did not develop soreness with arm movement, whatever preparation they received. No more than $27 \%$ of III-TT recipients developed redness or swelling at the injection site. When the latter reaction did occur, it was mild $(<3 \mathrm{~cm}$ in diameter) in the majority of cases and resolved completely within $72 \mathrm{~h}$ after vaccination. One recipient of III-TT had a temperature of $100.3^{\circ} \mathrm{F}$ that resolved within $24 \mathrm{~h}$. This fever was not believed to be attributable to vaccination since this subject also had other symptoms consistent with an upper res- piratory infection. No significant changes in blood chemistry values or $\mathrm{CBC}$ followed vaccination.

\section{CPS-specific antibody response}

The geometric mean concentration (GMC) of III CPS-specific IgG among the 30 recipients of the $58-\mu \mathrm{g}$ dose of III-TT rose from $0.09 \mu \mathrm{g} / \mathrm{ml}$ before immunization to $4.89 \mu \mathrm{g} / \mathrm{ml} 2$ wk thereafter (Table II). The level remained relatively unchanged for the next $6 \mathrm{wk}$ and was $3.02 \mu \mathrm{g} / \mathrm{ml}$ at $26 \mathrm{wk}$. Among the 30 recipients of III CPS, the GMC of specific IgG increased from $0.21 \mu \mathrm{g} / \mathrm{ml}$ before vaccination to a peak of $1.41 \mu \mathrm{g} / \mathrm{ml}$ at $8 \mathrm{wk}$. The GMC of III CPS-specific antibody was significantly higher 2 wk after immunization among recipients of the highest dose of III-TT than among recipients of III CPS $(P<0.05)$. Levels of specific antibody remained higher in the high-dose III-TT group than in the III CPS group at later time points, although the differences were not statistically significant. The relative immunogenicity of III-TT appeared to be dose dependent. The GMC of specific antibody among the 15 recipients of the $14.5-\mu \mathrm{g}$ dose of III-TT rose from $0.17 \mu \mathrm{g} / \mathrm{ml}$ before immunization to $2.31 \mu \mathrm{g} / \mathrm{ml} 2 \mathrm{wk}$ thereafter, with a peak level of $2.72 \mu \mathrm{g} /$ $\mathrm{ml} 8 \mathrm{wk}$ after immunization. Responses to the 3.6- $\mu \mathrm{g}$ dose were lower than those to higher doses of III-TT, with a mean peak level of $1.10 \mu \mathrm{g} / \mathrm{ml} 8 \mathrm{wk}$ after vaccination of the $15 \mathrm{recipi}$ ents. In fact, the GMCs for this group were similar to those for the group receiving III CPS. The prevaccination level of specific IgG in the placebo group $(0.15 \mu \mathrm{g} / \mathrm{ml})$ was not different from the prevaccination level in any other group and remained unchanged throughout the observation period. Each group of vaccine recipients had significantly higher GMCs of specific antibody than did placebo recipients at each interval after immunization (Table II).

The population distributions of specific antibody levels 8 wk after immunization with III-TT $(58 \mu \mathrm{g})$ or III CPS are shown in Fig. 1. Compared with III CPS, III-TT evoked a greater immune response, as evidenced by a shift to the right

Table II. Immunogenicity of Group B Streptococcal Vaccines in Healthy Women

\begin{tabular}{|c|c|c|c|c|c|c|}
\hline \multirow[b]{2}{*}{ Vaccine, dose } & \multirow{2}{*}{$\begin{array}{l}\text { No. of } \\
\text { recipients }\end{array}$} & \multicolumn{5}{|c|}{ GMC of type III CPS-specific IgG [95\% CI] (range) at indicated week after vaccination } \\
\hline & & 0 & 2 & 4 & 8 & 26 \\
\hline$\mu g$ & & & & $\mu g / m l$ & & \\
\hline \multicolumn{7}{|l|}{ III-TT } \\
\hline \multirow[t]{3}{*}{58} & 30 & $0.09^{*}$ & $4.89^{\ddagger \S}$ & $4.20^{\ddagger}$ & $4.53^{\ddagger}$ & $3.02^{\ddagger}$ \\
\hline & & {$[0.04-0.22]$} & [2.07-11.57] & {$[1.78-9.94]$} & {$[1.92-10.70]$} & [1.28-7.13] \\
\hline & & $(\leq 0.05-2.46)$ & $(\leq 0.05-367.36)$ & $(\leq 0.05-340.99)$ & $(0.07-325.12)$ & $(0.07-202.88)$ \\
\hline \multirow[t]{3}{*}{14.5} & 15 & $0.17 *$ & $2.31^{\ddagger}$ & $2.53^{\ddagger}$ & $2.72^{\ddagger}$ & $2.45^{\ddagger}$ \\
\hline & & {$[0.08-0.36]$} & {$[0.83-6.49]$} & [0.88-7.25] & [0.95-7.76] & [0.91-6.55] \\
\hline & & $(\leq 0.05-1.41)$ & $(\leq 0.05-95.17)$ & $(0.12-88.77)$ & $(0.19-98.30)$ & $(0.13-90.75)$ \\
\hline \multirow[t]{3}{*}{3.6} & 15 & $0.23^{*}$ & $0.91^{\ddagger}$ & $0.99^{\ddagger}$ & $1.10^{\ddagger}$ & $0.90^{\ddagger}$ \\
\hline & & [0.10-0.55] & [0.24-3.50] & [0.27-3.61] & [0.40-3.02] & {$[0.33-2.45]$} \\
\hline & & $(\leq 0.05-3.61)$ & $(\leq 0.05-76.29)$ & $(\leq 0.05-66.6)$ & $(0.06-28.99)$ & $(\leq 0.05-15.02)$ \\
\hline \multirow[t]{3}{*}{ III CPS, 50} & 30 & $0.21 *$ & $1.30^{\ddagger}$ & $1.40^{\ddagger}$ & $1.41^{\ddagger}$ & $1.20^{\ddagger}$ \\
\hline & & [0.09-0.49] & {$[0.55-3.10]$} & [0.59-3.33] & [0.59-3.37] & [0.50-2.86] \\
\hline & & $(\leq 0.05-20.45)$ & $(\leq 0.05-113.86)$ & $(\leq 0.05-110.08)$ & $(\leq 0.05-148.22)$ & $(\leq 0.05-151.94)$ \\
\hline \multirow[t]{3}{*}{ Saline, NA ${ }^{\|}$} & 10 & $0.15^{*}$ & 0.14 & 0.10 & 0.16 & 0.10 \\
\hline & & {$[0.05-0.43]$} & {$[0.04-0.44]$} & {$[0.02-0.46]$} & {$[0.06-0.45]$} & {$[0.02-0.42]$} \\
\hline & & $(\leq 0.05-1.36)$ & $(\leq 0.05-1.92)$ & $(\leq 0.05-1.63)$ & $(\leq 0.05-1.57)$ & $(\leq 0.05-1.25)$ \\
\hline
\end{tabular}

*NS, compared with one another. ${ }^{\ddagger} P<0.05$ versus saline. ${ }^{\S} P<0.05$ versus III CPS. ${ }^{\|}$Not applicable. 


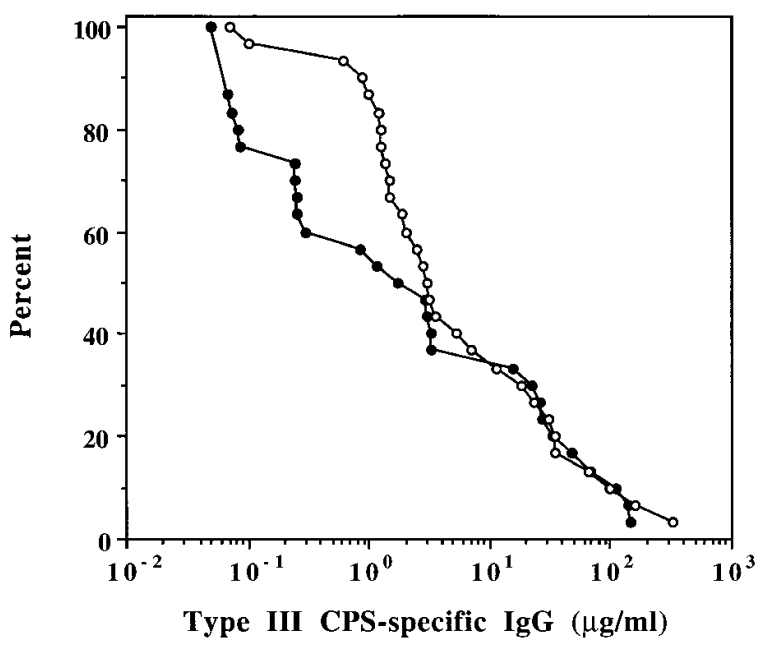

Figure 1. Reverse cumulative distribution plot of group B Streptococcus type III capsular polysaccharide-specific IgG level in women vaccinated with type III capsular polysaccharide-tetanus toxoid conjugate vaccine (open circles, $n=30$ ) or uncoupled type III capsular polysaccharide (solid circles, $n=30$ ).

of the upper portion of the III-TT distribution curve. For example, at this time point, $80 \%$ of III CPS recipients had III CPS-specific antibody levels $\geq 0.08 \mu \mathrm{g} / \mathrm{ml}$, while $80 \%$ of III-TT recipients had levels $\geq 1.24 \mu \mathrm{g} / \mathrm{ml}$.

\section{Proportional responses to III-TT and III CPS}

The geometric mean of individual rises (from before vaccination to $8 \mathrm{wk}$ afterward) in III CPS-specific antibody was 50.1fold for recipients of the $58-\mu \mathrm{g}$ dose of III-TT and 8.8 -fold for those receiving uncoupled III CPS. During the 26-wk observation period, the distribution of fold-rise values for subjects receiving III-TT overshadowed that of values for subjects receiving III CPS (repeated-measures Wilcoxon, $P=0.015$ ). Fold-rise distribution at 8 wk among recipients of III-TT was dose dependent, with geometric mean rises of 19.3 and 4.4, respectively, for recipients of $14.5-\mu \mathrm{g}$ and $3.6-\mu \mathrm{g}$ doses. Proportional response rates $8 \mathrm{wk}$ after vaccination were significantly different for the two preparations: $90 \%$ of III-TT recipients but only $50 \%$ of III CPS recipients had $\geq 4$-fold rises $(P=$ $0.0015)$.

\section{CPS-specific IgG subclasses, IgA and IgM}

IgG subclass-specific responses to III-TT $(n=9)$ and III CPS $(n=7)$ were measured. Median reciprocal $\mathrm{IgG}_{2}$ titer for III-TT recipients was 2,720 (range 445-15,020) and for III CPS recipients was 2,290 (range 630-12,450). Minimal or unmeasurable responses were seen to the other IgG subclasses: $\mathrm{IgG}_{1}$ (both groups, range $<50$ to 260 ), $\mathrm{IgG}_{3}$ (both groups, $<50$ to 80 ), and $\mathrm{IgG}_{4}$ (both groups, all $<50$ ).

Both GBS vaccines elicited higher specific IgA levels than saline. The GMC of III CPS-specific IgA among 15 recipients of high-dose III-TT rose from $0.13 \mu \mathrm{g} / \mathrm{ml}$ at week 0 to $1.02 \mu \mathrm{g} /$ $\mathrm{ml}$ at week 8. Similar responses were elicited by III CPS, with GMCs of 0.15 and $0.90 \mu \mathrm{g} / \mathrm{ml}$ at weeks 0 and 8 , respectively. The GMC of III CPS-specific IgA in the placebo group was $0.11 \mu \mathrm{g} / \mathrm{ml}$ at week 0 and $0.13 \mu \mathrm{g} / \mathrm{ml}$ at week 8 .

All 35 women enrolled in study 1 had undetectable levels of type-specific IgM before vaccination (titer, $<1: 50$ ). Only

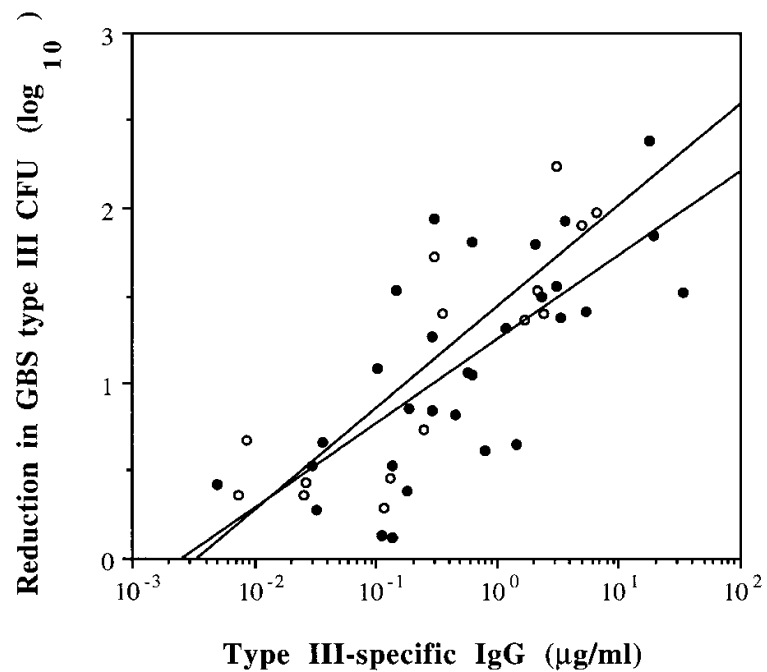

Figure 2. Relationship of in vitro opsonic activity and concentration of vaccine-induced type III group B Streptococcus capsular polysaccharide-specific IgG in sera from individuals vaccinated $4 \mathrm{wk}$ before with type III capsular polysaccharide-tetanus toxoid conjugate vaccine (solid circle, $r=0.71$ ) or uncoupled type III capsular polysaccharide (open circle, $r=0.85$ ). Killing is expressed as $\log _{10}$ reduction (on the y-axis) in CFU of GBS type III after incubation with the test serum, complement, and human blood leukocytes. Serum samples were tested at a 1:10 dilution, and the final assay concentration of type IIIspecific $\operatorname{IgG}$ is shown on the $\mathrm{x}$-axis.

three women had detectable type-specific IgM 8 wk after vaccination; the titers were 1:200 (III-TT group), 1:2,000 (III-TT group), and 1:300 (III CPS group) (13).

\section{Functional activity of vaccine-induced antibody}

In vitro. Sera obtained $4 \mathrm{wk}$ after vaccination were tested for functional activity in an opsonophagocytosis assay. Positive correlations of $r=0.71$ and 0.85 existed between the killing of GBS by polymorphonuclear leukocytes and the concentration of III CPS-specific IgG elicited to III-TT and III CPS, respectively (Fig. 2). Independent analysis of the correlation of antibody level with opsonophagocytic activity showed no difference between the slopes for III CPS and III-TT $(P>0.49)$ and showed virtually identical y-intercepts. The implication is that, at any given level of antibody, sera from individuals receiving either vaccine had similar functional capacity. This significant correlation also verified the ability of the ELISA to quantify functional antibodies.

In vivo. The functional activity of antibodies to III-TT was further examined by protection studies in mice. 30 (73\%) of 41 pups born to dams given an injection of standard human reference serum survived an ordinarily lethal GBS challenge, whereas all of 11 pups born to a dam given normal human serum died.

\section{Specificity of III-TT-induced antibodies}

The specificity of antibodies elicited by III-TT for a molecular size-dependent conformational epitope was examined by a comparison of their relative overall binding affinity for III CPS molecules of various molecular sizes in ELISA competition experiments $(11,21)$. In studies with one serum (Fig. 3), antibody binding was inhibited $50 \%$ by $1.6 \mathrm{nM}$ native III CPS $\left(M_{\mathrm{r}}>150,000\right)$. The $50 \%$ inhibitory concentration for III CPS of $\sim 25$ repeating units $\left(M_{\mathrm{r}}=25,500\right)$ was $1,300 \mathrm{nM}$; this 
Table III. Evaluation of Relative Affinities of GBS Type III Specific Antibodies Induced by GBS III-TT Conjugate Vaccine and GBS III Polysaccharide Vaccine

\begin{tabular}{cccc}
\hline & & \multicolumn{2}{c}{ IC $_{50}$ of native type III PS* } \\
\cline { 3 - 4 } Vaccine & Anti-GBS III IgG & Individual & Mean \pm SD $^{\ddagger}$ \\
\hline & $\mu g / m l$ & $n M$ & $n M$ \\
GBS III-TT & 3.0 & 1.8 & \\
& 3.5 & 2.9 & \\
& 5.3 & 1.2 & $2.2 \pm 0.8$ \\
& 15.1 & 2.0 & \\
GBS III & 50 & 3.0 & \\
& 0.8 & 1.7 & \\
& 6.4 & 3.6 & \\
& 17.5 & 2.5 & \\
& 21.6 & 0.05 & \\
& 94.2 & 2.2 & \\
& & &
\end{tabular}

* Concentration of GBS type III polysaccharide that inhibits $50 \%$ of the binding of sera in GBS III ELISA. * No significant difference.

result reflected a binding affinity almost 3 orders of magnitude lower than that for the native (high-molecular-weight) CPS. An oligosaccharide representing a single repeating unit of III CPS $\left(M_{\mathrm{r}}=1,022\right)$ inhibited antibody binding by $<25 \%$, even at concentrations as high as $125,000 \mathrm{nM}$. Similar affinity differences for saccharides of these three chain lengths were found for the other four III-TT postvaccination sera tested.

We compared the relative overall binding affinities of antibodies elicited by III-TT and III CPS in competitive ELISA inhibition experiments using the native III CPS as inhibitor. We selected five sera for each group representing a range of specific antibody concentrations $(3.0-50 \mu \mathrm{g} / \mathrm{ml}$ in III-TT-elicited sera and $0.8-94.2 \mu \mathrm{g} / \mathrm{ml}$ in III CPS-elicited sera). The mean $50 \%$ inhibitory concentration for III-TT sera was $2.2 \pm$ $0.8 \mathrm{nM}$ and for III CPS sera was $2.0 \pm 1.3 \mathrm{nM}$ (Table III). With the exception of a single III CPS serum, the $\mathrm{IC}_{50}$ values were between 1.1 and $3.0 \mathrm{nM}$.

Response to the TT component of III-TT

Among recipients of the $58-\mu \mathrm{g}$ dose of III-TT in study 1 , the GMC of TT-specific antibody was $35 \mu \mathrm{g} / \mathrm{ml}$ (range, 3.2-193 $\mu \mathrm{g} / \mathrm{ml}$ ) before vaccination and rose to $551 \mu \mathrm{g} / \mathrm{ml}$ (range, 306$1,440 \mu \mathrm{g} / \mathrm{ml}$ ) by $8 \mathrm{wk}$ after vaccination (Wilcoxon matched pairs, $P<0.001)$. No increases in TT antibody levels were noted in 8 -wk postvaccination sera from the 15 recipients of III CPS.

\section{Discussion}

Prevention of perinatal GBS infection has been identified as a national health priority (22). The impact of GBS infection on the health of newborns has prompted the Centers for Disease Control and Prevention $(23,24)$ and the American Academy of Pediatrics (25) to make specific recommendations concerning intrapartum antibiotic prophylaxis for perinatal GBS infection. The view shared by these groups is that such prophylaxis should be offered to women known to be vaginal carriers of GBS and should be recommended to those with risk factors predisposing to invasive disease. Antibiotic prophylaxis is not an ideal solution, however, because antibiotics may not prevent late-onset disease, may cause anaphylactic and other allergic reactions, and may lead to the emergence of resistant organisms $(23,24)$.

The best long-term solution appears to be active immunization (26). There are several reasons to believe that neonates and perhaps pregnant women can be protected from GBS disease by the administration of a CPS-based vaccine to women of childbearing age before or during pregnancy. First, although the minimal protective antibody concentration has not been determined precisely, the mothers of neonates developing GBS disease usually have very low levels of antibodies to the serotype-specific CPS of the organism infecting their respective children (7). Second, GBS CPS-specific IgG in the mother's serum crosses the placenta $(9,27)$. Third, infants receiving specific maternal IgG are protected from disease (28). Finally, nearly all infant disease develops within the first 3 mo of life (1), an interval during which transplacentally passed $\mathrm{IgG}$ can be found in infant sera.

The GBS type III glycoconjugate vaccine given to women of childbearing age, like the uncoupled III CPS vaccine, was well tolerated, with mild injection-site reactions noted in fewer than one-half of recipients. A dose-response study suggested that the highest dose of conjugate administered was superior to the two lower doses, although the differences were not statistically significant. By 2 wk after vaccination, the antibody re-

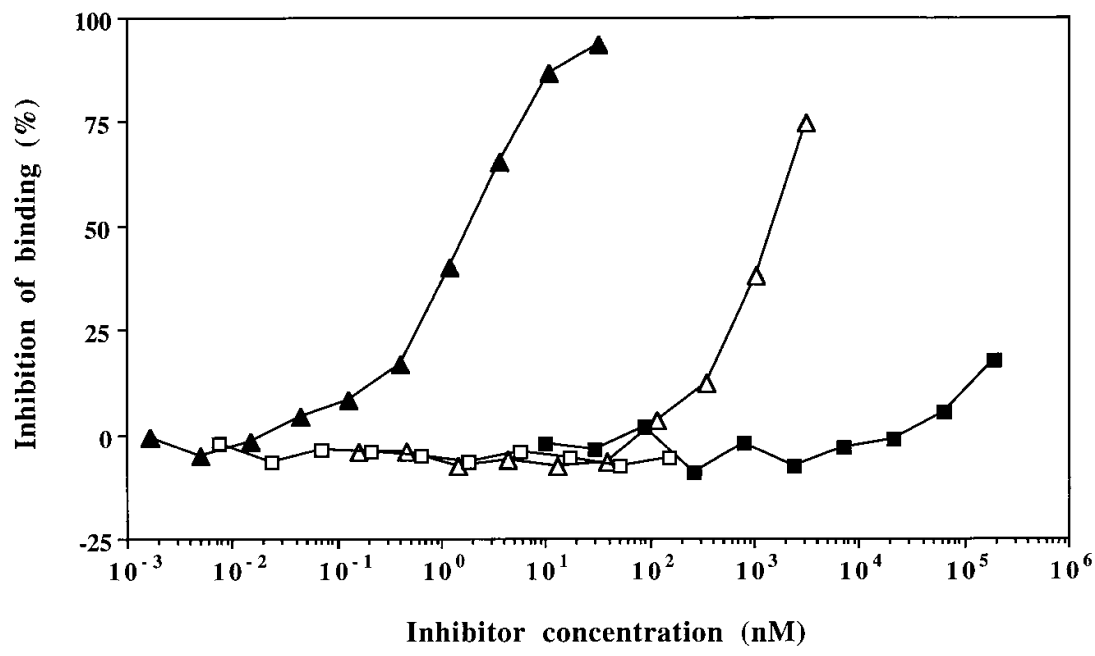

Figure 3. Epitope specificity of type III group B streptococcal antibodies in serum from recipient of type III capsular polysaccharide-tetanus toxoid conjugate vaccine. Symbols represent percent inhibition of antibody binding to type III-HSAcoated ELISA wells by various concentrations of type III CPS (closed triangles), 25-pentasaccharide-repeating-unit CPS (open triangles), 1-pentasaccharide-repeating-unit CPS (closed squares), and HSA (open squares). Each point is the average of duplicate determinations. 
sponse in the III-TT group had reached its maximal level. This rapid rise in antibody level is a clinically important characteristic of III-TT. If the vaccine were administered in the last trimester of pregnancy, a protective level of antibody would quickly be achieved. Antibodies transported by the placenta to the fetus may confer protective immunity even to infants born prematurely between 34 and 37 wk of gestation.

The postvaccination GMC of specific antibody among III-TT recipients was significantly greater than that among III CPS recipients 2 wk after immunization $(P<0.05)$. Despite persistently higher antibody levels in the III-TT group, the differences were not significant at subsequent time points. At each interval after immunization, both vaccines proved to be significantly more immunogenic than placebo. An important aspect of a vaccine's immunogenicity is the proportion of recipients who develop sufficient antibody to be protective against disease. In this regard, it may be useful to examine not only the mean antibody level for each group vaccinated, but also the distribution of individual antibody levels. Reverse cumulative distribution plots of 8-wk antibody concentrations of women receiving III-TT or III CPS demonstrated an advantage for the conjugate vaccine in that a larger proportion of III-TT vaccinees than of III CPS recipients had high antibody levels. Further, comparison of the proportions of individuals with potentially protective levels of antibody also reveals the superiority of III-TT vaccine. For example, 8 wk after immunization, $87 \%$ of III-TT recipients compared with only $53 \%$ of III CPS recipients had a theoretically protective antibody level of $1.0 \mu \mathrm{g} / \mathrm{ml}$ $(P=0.01)$. One method of estimating vaccine potency is by determining the percentage of recipients with fourfold or greater rises in antibody concentration. The proportion of recipients with a $\geq 4$-fold increase in specific antibody at $8 \mathrm{wk}$ after vaccination was higher for III-TT than for III CPS (90\% vs. $50 \% ; P=0.0015)$.

Antibodies elicited by immunization with the conjugate vaccine were primarily of the $\mathrm{IgG}$ class and the $\mathrm{IgG}_{2}$ subclass and displayed protective activity against GBS in vitro and in vivo. In opsonophagocytosis assays, III-TT-induced antibodies promoted killing of type III GBS by polymorphonuclear leukocytes in the presence of complement. The concentration of vaccine-induced III CPS-specific IgG directly correlated with the capacity of sera to mediate killing of GBS, whether elicited by III CPS or III-TT. This observation implies that despite chemical modification during the generation of the conjugate vaccine the CPS retained the labile epitopes required for induction of antibodies with the same functional capacity as those induced by the uncoupled native type III CPS. In addition, passive administration of antibodies from conjugate vaccine recipients to pregnant mice protected pups from lethal challenge with type III GBS. This result demonstrated the capacity of these antibodies to cross the placenta and to provide protection.

Oligosaccharides structurally similar to the single repeating unit of GBS type III CPS have been described on human glycoproteins (29). Although Hayrinen et al. (29) hypothesized cross-reactivity between GBS polysaccharides and human tissues, no cross-reactions were evident in immunobinding experiments conducted by these authors. The lack of such crossreactions may be explained by studies demonstrating that III CPS antibodies elicited in animals in response to killed GBS organisms or to III-TT recognize an epitope of III CPS whose conformation depends on the molecular size of the antigen
$(11,21)$. Thus, III CPS antibodies raised in rabbits bind with much higher affinity to III CPS of $M_{\mathrm{r}} \sim 98,000$ than to III CPS of smaller molecular size (11). The term affinity, in this context, denotes the overall strength of association of antibody and antigen, or "functional affinity" (30); functional affinity reflects the influence on binding strength of the multivalency of the antibody and antigen molecules as well as the intrinsic affinity of the antibody-combining site for its cognate epitope. Similarly, in the present study, antibodies in sera from III-TT recipients bound to full-length III CPS with much higher affinity (nearly 3 orders of magnitude) than to a 25-repeating-unit polysaccharide. The relative binding affinity of vaccine-induced antibodies for the 1-repeating-unit oligosaccharide was unmeasurable in these assays and was at least 5 orders of magnitude lower than that for full-length CPS. These results indicate that human antibodies evoked by III-TT recognize the molecular size-dependent conformational epitope of III CPS as it occurs on the bacterial surface; these antibodies bind weakly or not at all to small oligosaccharides of the same repeatingunit structure, such as those found on host tissues.

The induction in humans of functional antibodies directed towards a conformational epitope brings to fruition a model for vaccine design based on detailed knowledge of the immunochemical specificity of critical carbohydrate epitopes. Oxidation removed carbon 9 from a selected fraction of sialic acid residues on the polysaccharide and created an aldehyde group at the remaining eighth carbon. Prior nuclear magnetic resonance analysis has shown that this modification does not alter the conformational epitope of the polysaccharide (31). The type III CPS was coupled, by reductive amination, through the aldehyde groups to $\epsilon$-amino groups on lysine residues of the protein. This unique approach resulted in a conjugate vaccine with a preserved conformational epitope which elicited functionally active and protective antibodies. Furthermore, the design strategy of creating specific chemical sites for linkage of polysaccharides to proteins should result in vaccines with greater lot-to-lot reproducibility.

Experimental conjugates have been constructed with CPS of GBS serotypes Ia, Ib, II, III, and V (32-34). Each GBS conjugate has induced high levels of protective antibodies in animals and has proven more immunogenic than the homologous uncoupled CPS. Our initial clinical experience with the type III GBS conjugate vaccine provides evidence that vaccines of this design are well-tolerated and immunogenic. The delivery of GBS CPS conjugate vaccines through maternal immunization may be a realistic approach to the prevention of perinatal GBS infections.

\section{Acknowledgments}

The authors thank Dr. George Siber, Massachusetts State Biologics Laboratory, for measuring antibodies to tetanus toxoid; and Dr. Pamela McInnes, National Institute of Allergy and Infectious Diseases, for advice and assistance. Invaluable technical assistance in the production of vaccines was provided by April Blodgett and Julianne Pinel at the Channing Laboratory. Marcia A. Rench, Melissa E. Hickman, and Mary Hall at Baylor applied their expertise to the recruitment of subjects, collection of specimens, performance of assays and analysis of antibody responses.

This work was supported by National Institutes of Health, National Institute of Allergy and Infectious Diseases grant AI 30628, contract AI 25152, training grant T32 AI-07061 and the Edward and Amalie Kass Fellowship (H.-K. Guttormsen). 
This is a National Research Council of Canada publication (No. 39532).

The contents of this publication do not necessarily reflect the views or politics of the Department of Health and Human Services, nor does the mention of trade names, commercial products, or organizations imply endorsement by the U.S. government.

\section{References}

1. Baker, C.J., and M.S. Edwards. 1995. Group B streptococcal infections. In Infectious Diseases of the Fetus and Newborn Infant. J.S. Remington and J.O. Klein, editors. W.B. Saunders, Philadelphia, PA. 980-1054.

2. Farley, M.M., R.C. Harvey, T. Stull, J.D. Smith, A. Schuchat, J.D. Wenger, and D.S. Stephens. 1993. A population-based assessment of invasive disease due to group B Streptococcus in nonpregnant adults. N. Engl. J. Med. 328:1807-1811

3. Harrison, L.H., A. Ali, D.M. Dwyer, J.P. Libonati, M.W. Reeves, J.A. Elliott, L. Billmann, T. Lashkerwala, and J.A. Johnson. 1995. Relapsing invasive group B streptococcal infection in adults. Ann. Intern. Med. 123:421-427.

4. Jackson, L.A., R. Hilsdon, M.M. Farley, L.H. Harrison, A.L. Reingold, B.D. Plikaytis, J.D. Wenger, and A. Schuchat. 1995. Risk factors for group B streptococcal disease in adults. Ann. Intern. Med. 123:415-420.

5. Schuchat, A., K. Deaver-Robinson, B.D. Plikaytis, K. Zangwill, J. MohleBoetani, and J.D. Wenger. 1994. Multistate case-control study of maternal risk factors for neonatal group B streptococcal disease. Pediatr. Infect. Dis. J. 13: $623-629$

6. Lancefield, R.C., M. McCarty, and W.N. Everly. 1975. Multiple mouseprotective antibodies directed against group B streptococci: special reference to antibodies effective against protein antigens. J. Exp. Med. 142:165-179.

7. Baker, C.J., and D.L. Kasper. 1976. Correlation of maternal antibody deficiency with susceptibility to neonatal group B streptococcal infection. $N$. Engl. J. Med. 294:753-756.

8. Baker, C.J., and D.L. Kasper. 1985. Group B streptococcal vaccines. Rev. Infect. Dis. 7:458-467.

9. Baker, C.J., M.A. Rench, M.S. Edwards, R.J. Carpenter, B.M. Hays, and D.L. Kasper. 1988. Immunization of pregnant women with a polysaccharide vaccine of group B Streptococcus. N. Engl. J. Med. 319:1180-1220.

10. Jennings, H.J., and R.K. Sood. 1994. Synthetic glycoconjugates as human vaccines. In Neoglycoconjugates: Preparation and Applications. Y.C. Lee and R.T. Lee, editors. Academic Press/New York. 325-361.

11. Wessels, M.R., A. Munoz, and D.L. Kasper. 1987. A model of highaffinity antibody binding to type III group B Streptococcus capsular polysaccharide. Proc. Natl. Acad. Sci. USA. 84:9170-9174.

12. Wessels, M.R., L.C. Paoletti, D.L. Kasper, J.L. DiFabio, F. Michon, K. Holme, and H.J. Jennings. 1990. Immunogenicity in animals of a polysaccharide-protein conjugate vaccine against type III group B Streptococcus. J. Clin. Invest. 86:1428-1433.

13. Guttormsen, H.-K., C.J. Baker, M.S. Edwards, L.C. Paoletti, and D.L. Kasper. 1996. Quantitative determination of antibodies to type III group B streptococcal polysaccharide. J. Infect. Dis. 173:142-150.

14. Englund, J.A., W.P. Glezen, C. Turner, J. Harvey, C. Thompson, and G. Siber. 1995. Transplacental antibody transfer following maternal immunization with polysaccharide and conjugate Haemophilus influenzae type b vaccines. $J$. Infect. Dis. 171:99-105.

15. Wessels, M.R., and D.L. Kasper. 1989. Antibody recognition of the type 14 pneumococcal capsule. J. Exp. Med. 169:2121-2131.
16. Rodewald, A.K., A.B. Onderdonk, H.B. Warren, and D.L. Kasper. 1992. Neonatal mouse model of group B streptococcal infection. J. Infect. Dis. 166:635-639.

17. SAS Institute. 1990. SAS/STAT user's guide, version 6, volume 1. SAS Institute, Cary, $\mathrm{NC}$

18. Reed, G.F., B.D. Meade, and M.C. Steinhoff. 1995. The reverse cumulative distribution plot: a graphic method for exploratory analysis of antibody data. Pediatrics. 96:600-603.

19. Wei, L.J. 1985. Combining dependent tests with incomplete repeated measurements. Biometrika. 72:359-364.

20. Mehta, C.R. 1991. StatXact: a statistical package for exact nonparametric inference. Am. Statistician. 45:74-75.

21. Paoletti, L.C., D.L. Kasper, F. Michon, J. DiFabio, H.J. Jennings, T.D. Tosteson, and M.R. Wessels. 1992. Effects of chain length on the immunogenicity in rabbits of group B Streptococcus type III oligosaccharide-tetanus toxoid conjugates. J. Clin. Invest. 89:203-209.

22. Institutes of Medicine. 1985. Prospects for immunizing against Streptococcus group B. In New Vaccine Development: Establishing Priorities. Division of Health Promotion and Disease Prevention. Vol I. Diseases of importance in the United States. National Academy Press/Washington, DC. 424-438.

23. Schuchat, A., and J.D. Wenger. 1994. Epidemiology of group B streptococcal disease. Risk factors, prevention strategy, and vaccine development. $E p$ idemiol. Rev. 16:374-402.

24. Centers for Disease Control and Prevention. 1996. Prevention of perinatal group B streptococcal disease: a public health perspective. $M M W R .45$ (RR-7):1-25.

25. American Academy of Pediatrics, Committee on Infectious Diseases and Committee on Fetus and Newborn. 1992. Guidelines for prevention of group B streptococcal (GBS) infection by chemoprophylaxis. Pediatrics. 90: 775-778.

26. Mohle-Boetani, J.C., A. Schuchat, B.D. Plikaytis, J.D. Smith, and C.V. Broome. 1993. Comparison of prevention strategies for neonatal group B streptococcal infection. A population-based economic analysis. JAMA (J. Am. Med Assn.). 270:1442-1448.

27. Baker, C.J., D.L. Kasper, I.B. Tager, A. Paredes, S. Alpert, W.M. McCormack, and D. Goroff. 1977. Quantitative determination of antibody to capsular polysaccharide in infection with type III strains of group B Streptococcus. J. Clin. Invest. 59:810-818.

28. Baker, C.J., M.S. Edwards, and D.L. Kasper. 1981. Role of antibody to native type III polysaccharide of group B Streptococcus in infant infection. Pediatrics. 68:544-549.

29. Hayrinen, J., S. Pelkonen, and J. Finne. 1989. Structural similarity of the type-specific group B streptococcal polysaccharides and the carbohydrate units of tissue glycoproteins: evaluation of possible cross-reactivity. Vaccine. 7:217-224.

30. Hornick, C.L., and F. Karush. 1972. Antibody affinity. III. The role of multivalence. Immunochemistry. 9:325-340.

31. Jennings, H.J., C. Lugowski, and D.L. Kasper. 1981. Conformational aspects critical to the immunospecificity of the type III group B streptococcal polysaccharide. Biochem. 20:4511-4518.

32. Wessels, M.R., L.C. Paoletti, J. Pinel, and D.L. Kasper. 1995. Immunogenicity and protective activity in animals of a type $\mathrm{V}$ group B streptococcal polysaccharide-tetanus toxoid conjugate vaccine. J. Infect. Dis. 171:879-884.

33. Wessels, M.R., L.C. Paoletti, A.K. Rodewald, F. Michon, J. DiFabio, H.J. Jennings, and D.L. Kasper. 1993. Stimulation of protective antibodies against types Ia and Ib group B streptococci by a type Ia polysaccharide-tetanus toxoid conjugate vaccine. Infect. Immun. 61:4760-4766.

34. Paoletti, L.C., M.R. Wessels, F. Michon, J. DiFabio, H.J. Jennings, and D.L. Kasper. 1992. Group B Streptococcus type II polysaccharide-tetanus toxoid conjugate vaccine. Infect. Immun. 60:4009-4014. 